\title{
First-Rank Symptoms and Premorbid Adjustment in Young Individuals at Increased Risk of Developing Psychosis
}

\author{
Carmen Morcillo ${ }^{a, b}$ Jan Stochl ${ }^{d}$ Debra A. Russo ${ }^{a, b}$ Antonio Zambrana ${ }^{e}$ \\ Navanthi Ratnayake ${ }^{b}$ Peter B. Jones ${ }^{a-c}$ Jesus Perez ${ }^{a, b}$ \\ ${ }^{a}$ Department of Psychiatry, University of Cambridge, ${ }^{b}$ CAMEO Early Intervention in Psychosis Service, CPFT, and \\ 'NIHR Collaboration for Leadership in Applied Health Research and Care, Cambridge, and ${ }^{\mathrm{d}}$ Department of Health \\ Sciences, University of York, York, UK; e Department of Psychiatry, University of Salamanca, Salamanca, Spain
}

\section{Key Words}

At-risk mental state · Early intervention · First-rank symptoms · Premorbid adjustment · Psychosis · Risk . Schizophrenia

\begin{abstract}
Background: Individuals at clinical high risk (CHR) for psychosis represent a heterogeneous group with a high rate of comorbid psychiatric disorders. There is little information on whether certain qualitative aspects of psychotic symptoms among CHR individuals may be predictive of future psychosis. This study focused on describing the prevalence of firstrank symptoms (FRS) among a sample of CHR individuals and its association with future transition to psychosis and, from a neurodevelopmental perspective, the level of adjustment of individuals at CHR during their childhood was also analysed. Sampling and Methods: Participants comprised 60 individuals at CHR (according to the Comprehensive Assessment of At-Risk Mental States, (AARMS) at the time of their referral to an early intervention service and 60 healthy volunteers (HVs). All subjects were assessed by senior research clinicians using the Mini International Neuropsychiatric Interview (MINI), and the Positive and Negative Syndrome Scale (PANSS). FRS were defined according to Kurt Schnei-
\end{abstract}

der's original classification, and information was collected from PANSS, CAARMS and clinical reports. Early premorbid functioning was measured using the Premorbid Adjustment Scale (PAS). We grouped individuals by number and type of FRS and analysed transitions to full-blown psychosis over a 2-year follow-up period. We also correlated the general social and functional adjustment of these individuals during their childhood (6-11 years of age) with the future development of mental states at CHR and FRS. Results: Over $69 \%$ of CHR individuals had more than one DSM-IV psychiatric diagnosis, mainly within the affective and anxiety diagnostic spectra. At least one FRS was present in $43.3 \%$ of CHR individuals, and $21.6 \%$ of these had more than one. Auditory hallucinations and passivity experiences were the most commonly reported. Only $10 \%$ of individuals at CHR made a transition to first-episode psychosis (FEP) over 2 years and, except for passivity experiences, the presence of one or more FRS was not significantly associated with the transition to FEP. CHR individuals, especially those with FRS, had poorer premorbid functioning and adjustment as children across educational, social and peer relationship domains than HVs. However, this was not associated with FEP 2 years later. Conclusions: FRS might not be indicators of psychosis alone but of different psychiatric disorders. In line with the neurodevelopmental model of psychosis, individuals at CHR might

\begin{tabular}{ll}
\hline KARGER 125.s. & $\begin{array}{l}\text { () 2015 S. Karger AG, Basel } \\
0254-4962 / 15 / 0482-0120 \$ 39.50 / 0 \quad \text { Karger }\end{array}$ \\
$\begin{array}{l}\text { E-Mail karger@karger.com } \\
\text { www.karger.com/psp }\end{array}$ & $\begin{array}{l}\text { This is an Open Access article licensed under the terms of the } \\
\text { Creative Commons Attribution-NonCommercial 3.0 Un- } \\
\text { ported license (CC BY-NC) (www.karger.com/OA-license), } \\
\text { applicable to the online version of the article only. Distribu- } \\
\text { tion permitted for non-commercial purposes only. }\end{array}$
\end{tabular}

Jesus Perez

Block 7, Ida Darwin Site

Fulbourn Hospital

Fulbourn, Cambridge CB21 5EE (UK)

E-Mail jp440@ cam.ac.uk 
be exhibiting several vulnerability traits and manifestations of abnormal developmental processes that might predict a future psychiatric disorder and/or long-term impairment.

(c) 2015 S. Karger AG, Basel

\section{Introduction}

Individuals at clinical high risk (CHR) for psychosis represent a heterogeneous group where psychotic experiences are associated with a wide range of psychopathology, lacking the specificity and predictive validity to indicate a transition to psychosis $[1,2]$. Recent longitudinal studies among individuals at CHR have reported transition rates to full-blown psychosis of between 7 and 54\% [3]. While the clinical signs and symptoms among individuals at CHR have been widely studied at a dimensional level [4-6], few studies have evaluated possible qualitative aspects of positive psychotic symptoms that might predict poorer outcomes and/or conversion to psychosis [4].

Among positive symptoms, Kurt Schneider (18871967) defined 'first-rank symptoms' (FRS) as those that, despite not being pathognomonic, might have a decisive weight in differentiating schizophrenia from other mental disorders [7]. Further research has confirmed that FRS are not only common in schizophrenia $[8,9]$ but also in other severe non-schizophrenic [10] and affective psychoses [11]. Whereas discriminatory symptoms have not yet been found for individuals at $\mathrm{CHR}$, one question that remains unanswered relates to whether FRS, when present among CHR individuals, could be a negative indicator and predictor of future transition towards full-blown psychosis.

On the other hand, it has been widely documented that negative symptoms and, in particular, poor premorbid adjustment and functioning are early indicators of psychotic illness [12-14]. Several studies have reported low levels of functioning among individuals who are at CHR or during the premorbid phase of psychosis [14, 15]. However, from a developmental perspective, an issue that remains elusive is whether individuals at $\mathrm{CHR}$ and with FRS may present with a lower level of functioning at earlier stages of life, indicating a more severe developmental course over time.

Therefore, the goal of our study was 3-fold. Based on a sample of individuals at CHR who were referred to an early intervention in psychosis service and healthy volunteers (HVs) recruited from the same geographical area, we aimed to describe the following: (1) the prevalence of
FRS among individuals at CHR, (2) the association between FRS and transition to full-blown psychosis and (3) the level of adjustment of individuals at CHR and with FRS during their childhood (6-11 years of age) in terms of social and academic functioning.

\section{Methods}

We explored the presence of FRS among a sample of 60 individuals at $\mathrm{CHR}$ at the time of their referral to an early intervention service and $60 \mathrm{HVs}$. We grouped individuals by number and type of FRS and analysed transitions to full-blown psychosis over a 2 -year follow-up period. We then correlated the general social and functional adjustment of these individuals during their childhood (6-11 years of age) with the future development of mental states at CHR and FRS.

\section{Setting}

CAMEO (http://www.cameo.nhs.uk) is an early intervention in psychosis service offering management for people aged 14-35 years suffering from first-episode psychosis (FEP) in Cambridgeshire, UK. CAMEO also accepts referrals of people at CHR. Referrals are accepted from multiple sources, including general practitioners, other mental health services, school and college counsellors, relatives, and self-referrals [1].

\section{Sample}

A consecutive cohort of 60 help-seeking individuals (aged 1635 years) referred to CAMEO from February 2010 to September 2012 met the criteria for CHR, according to the Comprehensive Assessment of At-Risk Mental States (CAARMS) [16]. Referrals came to our offices via a number of different routes, including selfreferral, carers and relatives and schools and colleges, but mainly via primary care. All individuals identified as high risk for psychosis living and detected in Cambridgeshire and Peterborough were offered a systematic follow-up in the context of a prospective, naturalistic study called PAATH (Prospective Analysis of At-Risk Mental States and Transitions into Psychosis). Participants were followed up for 2 years from the initial referral date. During this period, they were asked to attend subsequent interviews where they completed structured interviews and questionnaires. In our sample, all individuals fulfilled the criteria for the attenuated psychotic symptoms group. In addition, 7 individuals (11.7\%) also qualified for the vulnerability traits group (individuals with a family history of psychosis in a first-degree relative or schizotypal personality disorder plus a 30\% drop in GAF score from premorbid level, sustained for a month, occurring within the previous 12 months or GAF score of $50 \%$ or less for the previous 12 months). Intake exclusion criteria were as follows: (1) acute intoxication or withdrawal associated with drug or alcohol abuse or any delirium, (2) confirmed intellectual disability (Wechsler Adult Intelligence Scale - tested IQ $<70$ ) or (3) prior total treatment with antipsychotics for more than 1 week.

During the same period (February 2010 to September 2012), a random sample of $60 \mathrm{HV}$ s was recruited by post, using the $\mathrm{PAF}^{\circledR}$ (Postal Address File) provided by Royal Mail, UK. To ensure that each CHR and HV resided in the same geographical location, 50 
corresponding postcodes, matching the first $4 / 5$ characters and digits of each recruited CHR participant (e.g. PE13 5; CB5 3), were randomly selected using Microsoft SQL Server, a relational database management system, in conjunction with the PAF database. Each of these 50 addresses was sent a recruitment flyer containing a brief outline of the study, inclusion criteria and contact details. If this failed to generate recruits, a consecutive sample of postcodes would be selected. This process was repeated until a match was recruited. An average of 100 flyers was sent to each postcode to recruit the $60 \mathrm{HVs}$. HVs interested in the study could only participate if they were aged 16-35 years, resided in the same geographical area as CHR participants (Cambridgeshire) and did not have previous contact with mental health services.

Ethical Approval

Ethical approval was granted by the Cambridgeshire East Research Ethics Committee.

\section{Measures}

All participants were assessed with sociodemographic (age, gender, ethnicity and occupational status) and several clinical measures at the time of their referral to CAMEO. The assessments were carried out by senior research clinicians trained in each of the measurement tools.

CHR participants were interviewed by senior trained psychiatrists working in CAMEO, using the Mini International Neuropsychiatric Interview (MINI), version 6.0.0 [17] - a brief structured diagnostic interview for DSM-IV Axis I psychiatric disorders. The Positive and Negative Syndrome Scale (PANSS) [18] for psychotic symptoms was employed to capture the severity of positive symptoms (7 items), negative symptoms ( 7 items) and general psychopathology (16 items) in a 7-point scale, with higher scores indicating greater severity of illness. Summary score and sub-domain scores of positive, negative and general psychopathology symptoms were computed. HVs were also assessed by senior researcher clinicians using the PANSS and CAARMS. All the assessments, including the CAARMS, were performed by the same assessor for each participant.

FRS were defined according to Kurt Schneider's original classification [7]. These included the following: (1) auditory hallucinations (hearing voices conversing with one another, voices heard commenting on one's actions and thought echo); (2) somatic hallucinations; (3) passivity experiences (delusions of control/being controlled); (4) thought withdrawal; (5) thought insertion; (6) thought broadcasting, and ( $\mathrm{g}$ ) delusional perceptions.

The existence and description of any of these symptoms was documented on the PANSS, CAARMS and clinical reports by experienced research clinicians working at CAMEO. The clinical assessments were supervised by senior consultant psychiatrists. For the purpose of this study, 2 blinded independent psychiatrists also gathered specific information related to FRS collected from the above-mentioned sources. Co-coding was then discussed with a senior consultant psychiatrist with expertise in psychosis.

The Premorbid Adjustment Scale (PAS) comprises 36 items describing levels of functioning before the onset of psychosis. These items cover sociability and withdrawal, peer relationships, scholastic performance, adaptation to school, and capacity to establish socio-sexual relationships, assessed during four periods in life: childhood (up to 11 years), early adolescence ( $12-15$ years), late adolescence (16-18 years), and adulthood (19 years and be- yond) [19]. The rating is based on interviews with the patient and/ or with family members. The scoring range of each item is $0-6$, with 0 indicating the best level of functioning and 6 the worst. We assessed premorbid functioning and adjustment during childhood (up to 11 years of age), and therefore the domain related to sociosexual relationships was not included. We obtained mean scores for each of the other four domains.

\section{Statistical Analysis}

Our primary method for comparison of categorical sociodemographic variables between individuals at $\mathrm{CHR}$ and HVs was Fisher's exact test. For age comparison the t test was used. We also employed Fisher's exact test to analyse associations between the presence of FRS in CHR individuals and transitions to psychosis. The Wilcoxon signed-rank test was used to compare PAS domains between CHR individuals and HVs. All computations were performed using R software [20].

\section{Results}

\section{Sociodemographic Profile}

Sociodemographic information was collected, comprising age, gender, ethnicity, and occupational status. Table 1 shows a comparison between CHR individuals and HVs. There was a difference in age between the two groups; HVs were significantly older than the CHR participants ( $\mathrm{p} \leq 0.001$ ). The CHR group had a slightly higher proportion of males and the HV group had a slightly higher proportion of females. Both groups were predominantly White with a similar proportion of mixed, Asian and Black participants. Both groups contained the same number of students (41.7\%), but significantly more HV participants were employed $(\mathrm{p}=0.001)$.

\section{DSM-IV Diagnoses and PANSS Scores}

We obtained MINI DSM-IV diagnoses for 55 of the 60 CHR individuals. Of these, 38 (69.1\%) had more than one DSM-IV psychiatric diagnosis, mainly within the affective and anxiety diagnostic spectra. Primary diagnoses for this group were ranked in terms of frequency, as follows: major depressive episode, current or recurrent $(n=26$; $47.3 \%)>$ social phobia $(n=7 ; 12.7 \%)=$ generalised anxiety disorder $(\mathrm{n}=7 ; 12.7 \%)>$ obsessive compulsive disorder $(\mathrm{n}=5 ; 9.1 \%)>$ bipolar disorder, type II $(\mathrm{n}=2 ; 3.6 \%)>$ panic disorder $(\mathrm{n}=1 ; 1.8 \%)=$ posttraumatic stress disorder $(\mathrm{n}=1 ; 1.8 \%)$. Overall, 6 CHR individuals (10.9\%) did not fulfil sufficient criteria for a DSM-IV Axis I diagnosis.

The mean PANSS scores for the CHR group comprised positive symptoms $(13.1, \mathrm{SD}=3.2)$, negative symptoms $(12.4, \mathrm{SD}=5.0)$ and general psychopathology $(32.7, \mathrm{SD}=7.0)$. These scores indicated a 'mildly ill' 
Table 1. Sociodemographic comparison between CHR individuals and HVs

\begin{tabular}{|c|c|c|c|}
\hline Sociodemographic characteristics & $\mathrm{CHR}(\mathrm{n}=60)$ & HVs $(n=60)$ & $\mathrm{p}$ values \\
\hline Age at study entry, years & & & $<0.001$ \\
\hline Median & 19.89 & 22.60 & \\
\hline Minimum & 16.41 & 16.18 & \\
\hline Maximum & 30.21 & 35.57 & \\
\hline \multicolumn{4}{|l|}{ Gender, $\mathrm{n}$} \\
\hline Female & $29(48.3 \%)$ & $34(56.7 \%)$ & 0.465 \\
\hline \multicolumn{4}{|l|}{ Ethnicity, $\mathrm{n}$} \\
\hline White & $56(93.3 \%)$ & $55(91.7 \%)$ & 1.000 \\
\hline Mixed & $2(3.3 \%)$ & $2(3.3 \%)$ & 1.000 \\
\hline Asian & $1(1.7 \%)$ & $2(3.3 \%)$ & 1.000 \\
\hline Black & $1(1.7 \%)$ & $1(1.7 \%)$ & 1.000 \\
\hline
\end{tabular}

White ethnicity: subjects who were White British, White Irish or other White backgrounds. Mixed ethnicity: subjects who were White and Black Caribbean, mixed White and Black African, mixed White and Asian, or any other mixed backgrounds. Asian ethnicity: subjects who were Indian or Chinese. Black ethnicity: subjects from any Black backgrounds.

Occupational status was broadly categorized into 3 groups. Unemployed: subjects who did not have a job - those either looking for work, not looking for work (e.g. housewife) or not able to work due to medical reasons. Employed: people with full/part-time employment or those who were employed but currently unable to work. Students: full/part-time students, including those also working some hours.

p values: Fisher's exact test, except for age comparison ( $t$ test). a Data on occupational status were missing for 7 participants. group with regards to psychotic symptoms [21]. PANSS scores for HVs were $7.1(\mathrm{SD}=0.4)$ for positive symptoms, $7.8(\mathrm{SD}=0.8)$ for negative symptoms and 16.4 $(\mathrm{SD}=1.3)$ for general psychopathology. None of the HVs fulfilled CAARMS criteria for CHR.

\section{Frequency of FRS and Associations with Transitions}

The presence of any or more than one FRS was significantly higher among those individuals at CHR compared to HVs, who did not report FRS in our sample ( $\mathrm{p}<$ $0.001)$. A total of 26 individuals at CHR (43.3\%) presented at least one FRS, and $21.6 \%$ of them $(n=13)$ presented more than one. Among the different FRS, auditory hallucinations (mainly voices conversing with one another or commenting on one's actions) were the most frequently reported $(28.3 \%)$ followed by passivity experiences (25\%), thought broadcasting (10\%), thought insertion (8.3\%), and thought withdrawal (5\%). Individuals at CHR in our sample did not report any somatic hallucination or delusional perception (fig. 1).

In our sample, only $6(10 \%)$ individuals at CHR made a transition to FEP over 2 years, according to the

First-Rank Symptoms and Risk for Psychosis

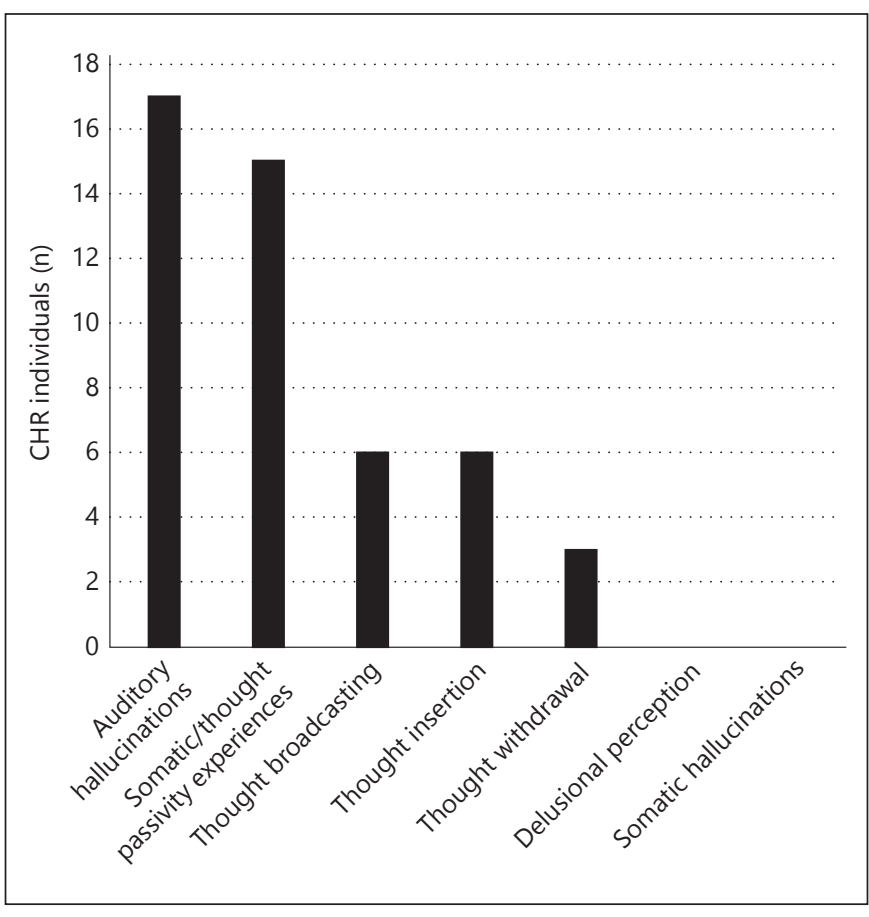

Fig. 1. Distribution and frequency of FRS in CHR individuals. 


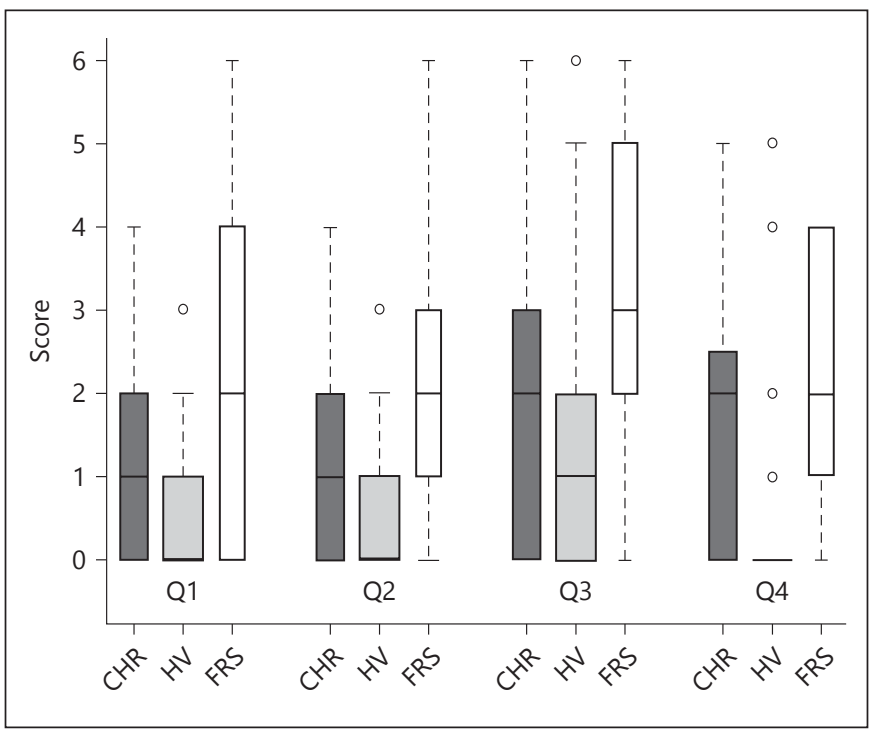

Fig. 2. Comparison of PAS domains (6-11 years of age) between CHR individuals, HVs and a subgroup of CHR individuals with FRS.

CAARMS. With regards to FRS, the presence of one or more than one was not significantly associated with transition to FEP ( $\mathrm{p}=0.388$ and $\mathrm{p}=0.109$, respectively). However, when taking into account each FRS individually, a statistical significant association was found between passivity experiences and later transition to psychosis $(\mathrm{p}=0.029)$.

\section{Early Functioning of Individuals at CHR and}

Experiencing FRS

Figure 2 shows the comparison of the mean scores between HVs and CHR individuals in the domains of the PAS. A third subgroup formed by those individuals at CHR who presented with FRS is also included.

There were statistically significant differences across the four domains - sociability and withdrawal $(p<0.001)$, peer relationships $(\mathrm{p}<0.001)$, scholastic performance $(\mathrm{p}=0.002)$, and adaptation to school $(\mathrm{p}<0.001)$. CHR individuals disclosed lower levels of adjustment and functioning when they were children compared to HVs. In our sample, even poorer levels were reported among those CHR individuals with FRS. In fact, CHR individuals with FRS reported significantly poorer adjustment in the PAS total score $(\mathrm{p}=0.024)$, specifically for peer relationships $(\mathrm{p}=0.024)$ and scholastic performance $(\mathrm{p}=0.046)$, than those at CHR but without FRS. However, no differences were found between individuals at $\mathrm{CHR}$ who had made transition to FEP 2 years later and those who did not.

\section{Discussion}

Our study, based on a sample of 60 help-seeking CHR individuals and $60 \mathrm{HVs}$, aimed to describe the prevalence of FRS among CHR individuals, their possible predictive value to future transition to psychosis and the early levels of adjustment and functioning among $\mathrm{CHR}$ individuals. More specifically, our findings were as follows: (1) the prevalence of at least one FRS among CHR individuals was over $43 \%$ and over $20 \%$ for more than one FRS, (2) among all FRS, only passivity experiences were associated with future transition to psychosis and (3) individuals at CHR presented significantly lower levels of functioning and adjustment during their childhood across educational, social and peer relationship domains, with those who reported FRS being even more affected. However, this did not predict future transition to psychosis.

FRS have been considered non-understandable psychological phenomena, non-culture dependent and, probably, the essence of schizophrenia [7, 22]. However, the diagnostic specificity of schneiderian FRS for schizophrenia has long been challenged, and a number of studies have called into question the continuous emphasis on bizarre delusions and special types of hallucinations (such as hearing voices conversing with one another or voices heard commenting on one's actions) in diagnostic classifications [23]. Indeed, FRS appear to be highly prevalent in the whole spectrum of functional psychotic disorders, including affective psychoses, where the prevalence of FRS has been reported to range between 22 and 29\% [10, 11, 24]. Therefore, FRS may lack discriminatory diagnostic value among psychotic disorders [23].

Notably, we also found a high prevalence of FRS in young individuals at increased risk of developing psychosis. However, their presence was not indicative of conversion to psychotic disorders. With the exception of passivity phenomena, which were among the most prevalent FRS in our sample, none of the schneiderian FRS showed a clear association with potential transitions. This finding is in line with previous empirical phenomenological studies that have described the decreased and disturbed 'sense of self-presence' as a core feature in schizophrenia and its prodromal phase, involving different and vague self-perceptions such as depersonalization, somatic disturbances and feelings of identity loss $[25,26]$.

Overall, our results are in agreement with previous research supporting the view that FRS should be considered symptoms of psychosis rather than symptoms of schizo- 
phrenia $[9,11,24]$. However, the low transition rates and the fact that most CHR individuals suffered from mood and anxiety disorders suggest that FRS should not only be considered markers for psychotic disorders but also for a wider range of mental disorders, which may present with psychotic experiences that may not evolve to frank psychotic disorders $[4,27,28]$.

These findings might support the dimensional phenotypic classification that is being proposed for schizophrenia. According to the neurodevelopmental model of schizophrenia, psychotic illness would be at the end of a spectrum of abnormal neurodevelopmental processes that begin years before the onset of illness [29]. These processes, resulting from different genetic [30], obstetric [31] and environmental factors [32], might not be specific predictors of schizophrenia alone but of a wide range of disorders and future clinical need [29]. Supporting the view of a neurodevelopmental perspective, our study showed that those individuals with poorer functioning and adjustment as children across educational, peer relationship and social domains might eventually develop psychotic symptoms as young adults, and that there was a dose-response relationship between poor premorbid adjustment and presence of FRS. Although not associated with later transition to full-blown psychosis in our sample, poor functioning and adjustment from early stages in development might be indicative of some vulnerability traits in these individuals to experience psychotic symptoms in the future, if they were not emerging already, as well as other non-psychotic mental health problems. It is unclear which aspects of poor functioning and adjustment in childhood might specifically predict one disorder over another. There is some evidence from longitudinal studies that certain receptive language, communication and cognitive deficits in childhood might specifically be associated with future psychosis $[5,33]$, whereas deficits in emotional and social/interpersonal development might be common predictors of psychosis, depression and bipolar and anxiety disorders [33]. Accordingly, poor premorbid adjustment might be the earliest manifestation of a common neurodevelopmental pathway for different psychiatric disorders and/or functional impairment [3436]. Further understanding and early intervention at these stages might be helpful to prevent future negative outcomes.

Our study has several strengths. For example, it was controlled, including both HVs and help-seeking CHR individuals. Also, its longitudinal design and high retention rates over 2 years allowed us to address the limitations associated with cross-sectional studies. However, our results should be considered in the light of some limitations. Our sample size did not allow further adjustment for comorbid mental disorders, which may have shed light on specific associations between the level of impairment and increased risk for non-psychotic mental disorders. Studies with larger samples will also be required in order to replicate findings regarding associations between specific FRS and future conversions to psychosis, especially the relevance of those FRS that were absent in our sample (somatic hallucinations and delusional perceptions). Early premorbid adjustment was measured retrospectively, bringing the possibility of recall bias. Finally, as transitions to psychosis were described in a 2-year follow-up period, it is possible that conversion rates could have been higher if follow-up had been longer.

\section{Acknowledgements}

This work was supported by the National Institute for Health Research (NIHR) - programme grant RP-PG-0606-1335 'Understanding Causes and Developing Effective Interventions for Schizophrenia and Other Psychoses' (awarded to P.B.J.). The work forms part of the NIHR Collaboration for Leadership in Applied Health Research and Care for Cambridgeshire and Peterborough (CLAHRC-CP). The NIHR had no further role in the study design, the collection, analysis and interpretation of data, the writing of the report, and the decision to submit the paper for publication.

The authors thank the PAATH Study team (Michelle Painter, Gillian Shelley, Chris McAlinden, Carolyn Crane, and Gerhard Smith) and all members of CAMEO services for their help and support in the execution of this study.

\section{Disclosure Statement}

The authors have no conflicts of interest based on business relationships of their own or of immediate family members.

References

Hui C, Morcillo C, Russo DA, Stochl J, Shelley
GF, Painter M, Jones PB, Perez J: Psychiatric
morbidity, functioning and quality of life in
young people at clinical high risk for psycho-
sis. Schizophr Res 2013;148:175-180.
Salokangas RK, Ruhrmann S, von Reventlow
HG, Heinimaa M, Svirskis T, From T, Luu-
tonen S, Juckel G, Linszen D, Dingemans P,
Birchwood M, Patterson P, Schultze-Lutter F,
Klosterkotter J: Axis I diagnoses and transi-
tion to psychosis in clinical high-risk patients
EPOS project: prospective follow-up of 245
clinical high-risk outpatients in four coun-
tries. Schizophr Res 2012;138:192-197.


-3 Correll CU, Hauser M, Auther AM, Cornblatt BA: Research in people with psychosis risk syndrome: a review of the current evidence and future directions. J Child Psychol Psychiatry 2010;51:390-431.

4 Armando M, Nelson B, Yung AR, Saba R, Monducci E, Dario C, Righetti V, Birchwood M, Fiori NP, Girardi P: Psychotic experience subtypes, poor mental health status and helpseeking behaviour in a community sample of young adults. Early Interv Psychiatry 2012;6: 300-308.

-5 Bearden CE, Wu KN, Caplan R, Cannon TD: Thought disorder and communication deviance as predictors of outcome in youth at clinical high risk for psychosis. J Am Acad Child Adolesc Psychiatry 2011;50:669-680.

-6 Smeets F, Lataster T, van WR, de GR, Ten $\mathrm{HM}$, Van OJ: Testing the hypothesis that psychotic illness begins when subthreshold hallucinations combine with delusional ideation. Acta Psychiatr Scand 2013;127:34-47.

7 Schneider K: Clinical Psychopathology. New York, Grune \& Stratton, 1959.

8 Carpenter WT Jr, Strauss JS: Cross-cultural evaluation of Schneider's first-rank symptoms of schizophrenia: a report from the International Pilot Study of Schizophrenia. Am J Psychiatry 1974;131:682-687.

-9 Rosen C, Grossman LS, Harrow M, BonnerJackson A, Faull R: Diagnostic and prognostic significance of Schneiderian first-rank symptoms: a 20-year longitudinal study of schizophrenia and bipolar disorder. Compr Psychiatry 2011;52:126-131.

10 Peralta V, Cuesta MJ: Diagnostic significance of Schneider's first-rank symptoms in schizophrenia. Comparative study between schizophrenic and non-schizophrenic psychotic disorders. Br J Psychiatry 1999;174: 243-248.

-11 Ihara K, Morgan C, Fearon P, Dazzan P, Demjaha A, Lloyd T, Kirkbride JB, Hayhurst H, Murray RM, Jones PB: The prevalence, diagnostic significance and demographic characteristics of Schneiderian first-rank symptoms in an epidemiological sample of firstepisode psychoses. Psychopathology 2009; 42 81-91.

12 Larsen TK, McGlashan TH, Johannessen JO, Vibe-Hansen L: First-episode schizophrenia. II. Premorbid patterns by gender. Schizophr Bull 1996;22:257-269.

13 Addington J, Addington D: Patterns of premorbid functioning in first episode psychosis: relationship to 2-year outcome. Acta Psychiatr Scand 2005;112:40-46.
14 Addington J, Penn D, Woods SW, Addington D, Perkins DO: Social functioning in individuals at clinical high risk for psychosis. Schizophr Res 2008;99:119-124.

15 Grano N, Karjalainen M, Suominen K, Roine M: Poor functioning ability is associated with high risk of developing psychosis in adolescents. Nord J Psychiatry 2011;65:16-21.

16 Yung AR, Yuen HP, McGorry PD, Phillips LJ, Kelly D, Dell'Olio M, Francey SM, Cosgrave EM, Killackey E, Stanford C, Godfrey K, Buckby J: Mapping the onset of psychosis: the Comprehensive Assessment of At-Risk Mental States. Aust NZ J Psychiatry 2005;39:964-971.

17 Sheehan DV, Lecrubier Y, Sheehan KH, Amorim P, Janavs J, Weiller E, Hergueta T, Baker R, Dunbar GC: The Mini-International Neuropsychiatric Interview (M.I.N.I.): the development and validation of a structured diagnostic psychiatric interview for DSM-IV and ICD-10. J Clin Psychiatry 1998; 59(suppl 20):22-33.

18 Kay SR, Fiszbein A, Opler LA: The Positive and Negative Syndrome Scale (PANSS) for schizophrenia. Schizophr Bull 1987;13:261-276.

19 Larsen TK, Friis S, Haahr U, Johannessen JO, Melle I, Opjordsmoen S, Rund BR, Simonsen E, Vaglum PV, McGlashan TH: Premorbid adjustment in first-episode non-affective psychosis: distinct patterns of pre-onset course. Br J Psychiatry 2004;185:108-115.

20 R Development Core Team: R: A language and environment for statistical computing. Vienna, R Foundation for Statistical Computing, 2014.

21 Leucht S, Kane JM, Kissling W, Hamann J, Etschel E, Engel RR: What does the PANSS mean? Schizophr Res 2005;79:231-238.

22 Jaspers K: Clinical Psychopathology. Chicago, University of Chicago, 1963

-23 Tandon R, Gaebel W, Barch DM, Bustillo J, Gur RE, Heckers S, Malaspina D, Owen MJ, Schultz S, Tsuang M, Van OJ, Carpenter W: Definition and description of schizophrenia in the DSM-5. Schizophr Res 2013;150:3-10.

24 Nordgaard J, Arnfred SM, Handest P, Parnas $\mathrm{J}$ : The diagnostic status of first-rank symptoms. Schizophr Bull 2008;34:137-154.

25 Moller P, Husby R: The initial prodrome in schizophrenia: searching for naturalistic core dimensions of experience and behavior. Schizophr Bull 2000;26:217-232.

-26 Parnas J, Handest P, Saebye D, Jansson L: Anomalies of subjective experience in schizophrenia and psychotic bipolar illness. Acta Psychiatr Scand 2003;108:126-133.

27 Van OJ, Linscott RJ, Myin-Germeys I, Delespaul P, Krabbendam L: A systematic review and meta-analysis of the psychosis continuum: evidence for a psychosis proneness-persistence-impairment model of psychotic disorder. Psychol Med 2009;39:179-195.
28 Yung AR, Nelson B, Baker K, Buckby JA, Baksheev G, Cosgrave EM: Psychotic-like experiences in a community sample of adolescents: implications for the continuum model of psychosis and prediction of schizophrenia. Aust NZ J Psychiatry 2009;43:118-128.

29 Rapoport JL, Giedd JN, Gogtay N: Neurodevelopmental model of schizophrenia: update 2012. Mol Psychiatry 2012;17:1228-1238.

30 Raznahan A, Greenstein D, Lee Y, Long R, Clasen L, Gochman P, Addington A, Giedd JN, Rapoport JL, Gogtay N: Catechol-omethyl transferase (COMT) val ${ }^{158}$ met polymorphism and adolescent cortical development in patients with childhood-onset schizophrenia, their non-psychotic siblings, and healthy controls. Neuroimage 2011;57: 1517-1523.

-31 Clarke MC, Tanskanen A, Huttunen M, Leon DA, Murray RM, Jones PB, Cannon M: Increased risk of schizophrenia from additive interaction between infant motor developmental delay and obstetric complications: evidence from a population-based longitudinal study. Am J Psychiatry 2011;168:1295-1302.

32 Van OJ, Kenis G, Rutten BP: The environment and schizophrenia. Nature 2010;468: 203-212.

33 Cannon M, Caspi A, Moffitt TE, Harrington H, Taylor A, Murray RM, Poulton R: Evidence for early-childhood, pan-developmental impairment specific to schizophreniform disorder: results from a longitudinal birth cohort. Arch Gen Psychiatry 2002;59: 449-456.

- 34 Carrion RE, McLaughlin D, Goldberg TE, Auther AM, Olsen RH, Olvet DM, Correll $\mathrm{CU}$, Cornblatt BA: Prediction of functional outcome in individuals at clinical high risk for psychosis. JAMA Psychiatry 2013;70:11331142.

35 Carrion RE, Goldberg TE, McLaughlin D, Auther AM, Correll CU, Cornblatt BA: Impact of neurocognition on social and role functioning in individuals at clinical high risk for psychosis. Am J Psychiatry 2011;168:806813.

-36 Salokangas RK, Heinimaa M, From T, Löyttyniemi E, Ilonen T, Luutonen S, Hietala J, Svirskis T, von Reventlow HG, Juckel G, Linszen D, Dingemans P, Birchwood M, Patterson P, Schultze-Lutter F, Ruhrmann S, Klosterkötter J; EPOS group: Short-term functional outcome and premorbid adjustment in clinical high-risk patients. Results of the EPOS project. Eur Psychiatry 2014;29: 371-380. 